\title{
A Pilot Study on the Effectiveness of a Heat-Pain Stimulus to induce Pain, Anxiety, and Fear
}

\section{Original Paper}

While the majority of previous studies assessing pain-related variables in psychopaths used electric shocks, little is known about the effectiveness of alternative pain-inducing methods to increase emotional responses such as fear and anxiety. A small sample of healthy undergraduate men $(\mathrm{N}=15)$ was recruited to assess the effectiveness of a heat stimulus to induce pain in an immediate versus delayed punishment paradigm. Although pain catastrophizing, anxiety, and threat of pain did not increase throughout the experiment, participants experienced a significant increase of fear of pain and pain intensity, indicating that the heat stimulus was effective in inducing pain. Furthermore, subjects were slower in initiating the pain stimulus during the first five trials, but no time difference was found during the 15 remaining trials. No correlation was found between psychopathic traits and pain-related variables, with the exception of inconsistent results within the Fearless Dominance factor. Findings are discussed in terms of improvement for a larger scale study involving psychopathic individuals.

Keywords: Heat-Pain, Pilot Study, Anxiety, Fear, Psychopathy

Guillaume Durand

Research Master in Cognitive and Clinical Neuroscience Alumni, specialization in Psychopathology

gdurao61@uottawa.ca 


\section{DURAND}

\section{INTRODUCTION}

Psychopathy is a personality disorder characterized by a lack of empathy, callousness, manipulative behavior, impulsivity, and a parasitic lifestyle (Berg et al., 2013; Seibert, Miller, Few, Zeichner, \& Lynam, 2011). Although not inherent components of the construct, several traits such as lower levels of anxiety, greater fear immunity, along with greater pain tolerance, are moderately associated with psychopathy (Berg et al., 2013; Miller, Rausher, Hyatt, Maples, \& Zeichner, 2014; Skeem \& Cooke, 2010). However, results regarding these associated constructs are highly mitigated, as some researchers refute the existence of a correlation between psychopathy and constructs such as anxiety and pain due to the lack of evidence found in their respective studies (Fedora \& Reddon, 1993; Visser, Ashton, \& Pozzebon, 2012).

This discrepancy across conclusions regarding the association of pain and psychopathy may stem from multiple sources, such as the instruments used to induce pain within psychopaths and non-psychopaths, and the type of population used, such as inmates and healthy individuals from the community. Early experiments investigating the relationship between pain and psychopathy were performed in male inmates by the use of electric shocks, and held similar results to one another (Hare \& Thorvaldson, 1970; Hare, 1965, 1966, 1968). These previous studies support that, while receiving painful electric shocks, psychopaths are not conditioned to fear pain, are not threatened by the fear of pain, are willing to receive higher levels of pain when incentives such as cigarettes are used, and report an overall lower level of pain than non-psychopaths.

A subsequent study has identified a link between psychopathy and the choice of immediate versus delayed punishment (Hare, 1966). Results suggest an absence of threat of pain in psychopaths, who were unable to develop any fear conditioning over time. Alternatively, non-psychopaths experienced more fear, which prompted behavioral responses to face the pain stimulus as soon as possible. When given the option of receiving a strong electric shock either immediately or in 10 seconds, $50 \%$ of the psychopaths opted for the immediate shock, while $67 \%$ of non-psychopaths opted for immediate shock during the first trial. Over the course of the trials, non-psychopaths developed a higher preference for the immediate shock, rising to a total of $82.3 \%$. However, preferences remained constant for psychopaths throughout the experiment. This study demonstrates the effectiveness of electric shocks to induce differential behavioral responses in psychopathic and non-psychopathic individuals. 
Fedora and Reddon (1993) challenged the hypothesis that psychopaths are more difficult to condition using fear of pain. They investigated the tolerance level to electrical stimulation within psychopathic and non-psychopathic inmates along with a control group composed of healthy male volunteers from the community. They concluded that, although both inmate groups could tolerate higher levels of pain than the control group, the tolerance did not differ between psychopathic and non-psychopathic inmates. The authors attributed these results to differential learning in inmates, as incarcerated populations face harsher treatment in everyday life than non-incarcerated individuals.

Due to the inconsistent findings of previous research performed with male inmates by the use of an electric shocking device, a subsequent study was performed on 104 individuals from the community (Miller et al., 2014). The purpose of this study was to determine the effectiveness of inducing pain and behavioral responses with alternative pain stimuli in psychopaths. Pain tolerance in response to an algometer, a cold pressor, and electrical stimulation was compared to the level of psychopathic traits. Results highlighted weak but significant correlations between the expression of psychopathic personality traits and pain tolerance to pressure $(\mathrm{r}=.27)$ and electric shock $(\mathrm{r}=.25)$, but not tolerance to cold pressor $(\mathrm{r}=$ .05). Although results were not conclusive regarding the use of a cold pressor, the significant correlation of psychopathic trait expression with pressure supports the possibility of assessing the fear of pain response with respect to psychopathy with an alternative and untested paininducing device, such as a heat-inducing device.

To our knowledge, no previous study has used a heat stimulus in psychopathy-related research. Considering the lack of previous data on the effectiveness of a heat stimulus in inducing pain in psychopaths, a preliminary study in a healthy sample is needed to assess its effectiveness before engaging in a costly and time consuming larger scale study. Indeed, due to the weak correlations found between pain tolerance and psychopathy, and the difficulty of recruiting large samples of psychopaths, a healthy sample is necessary for a preliminary study in order to ensure the stimulus' variability during the experiment in order to limit potential ceiling effects (Miller et al., 2014). A lack of variability within behavioral responses during an experiment within a healthy sample would reduce the possibility to detect differences when compared to psychopaths. Considering every individual possesses psychopathic traits to different degrees, and that psychopaths are at the far end of the continuum of psychopathic traits, a pilot study in a healthy sample may be sufficient to provide preliminary results regarding the correlation between higher psychopathic traits and response towards fear of pain variables (Berg et al., 2013). Hence, the purpose of this pilot study is to establish a paradigm for 
a future study assessing how psychopathic individuals react towards pain in terms of fearrelated constructs, such as anxiety, pain catastrophizing, and threat. Furthermore, the design of the current study should take fear conditioning of psychopaths into consideration. Based on previous work (Hare, 1966), participants were given the choice to start a pain stimulus immediately or after a 10 second countdown over the course of 20 trials. After every 5 trials, participants' perception of fear-related variables such as fear of pain, pain intensity, and threat of pain was assessed. The temperature was set to increase after every trial in order to reduce habituation and to increase fear of pain.

An increase of anxiety, pain catastrophizing, fear of pain, pain intensity, and threat of pain over the course of the experiment was expected. It was also hypothesized that participants would display an increase of preference to start the pain stimulus faster over the course of the experiment. Confirmation of these two hypotheses would support the effectiveness of the experimental design to use heat as a pain stimulus in psychopathy research. Lastly, a negative correlation between psychopathic traits and fear-related variables was expected alongside a positive correlation between psychopathic traits and the time to initiate the pain stimulus.

\section{METHODS}

\section{Participants}

A total of 15 male undergraduates from Maastricht University was recruited and gave written informed consent to take part in this experiment. This study was approved by the Ethical Committee of Psychology from Maastricht University. The participants' ages ranged between 19 and 28 years $(M=23.20, S D=2.91)$. Due to the preliminary nature of the study, no further inclusion or exclusion criteria were applied.

\section{Instruments}

Self-report measures

Psychopathic personality traits 


\section{DURAND}

The Psychopathic Personality Inventory-Short Form (PPI-SF) is a 56-item self-report questionnaire and is derived from the original 187-item PPI (Lilienfeld \& Widows, 2005). It assesses eight psychopathic traits, namely Machiavellian Egocentricity, Social Potency, Fearlessness, Coldheartedness, Impulsive Nonconformity, Blame Externalization, Carefree Nonplanfulness and Stress Immunity. The questionnaire is rated on a 4-point Likert scale ranging from 1 ('False') to 4 ('True'). The PPI-SF is divided into two factors: Fearless Dominance (FD), which includes Social Potency, Fearlessness and Stress Immunity, and Impulsive Antisociality (IA), which includes Machiavellian Egocentricity, Impulsive Nonconformity, Blame Externalization, and Carefree Nonplanfulness. This questionnaire is considered to be highly consistent, with Cronbach's alpha ranging from $\alpha=.70$ to .94 (Cale \& Lilienfeld, 2006; Mullins-Nelson, Salekin, \& Leistico, 2006; Tonnaer, Cima, Sijtsma, Uzieblo, \& Lilienfeld, 2013). The internal consistency of the PPI-SF in the present study was fairly low ( $\alpha=$ .29), which is not surprising as two opposite constructs (FD and IA) were being assessed in a small sample. Examination of internal consistency by alpha revealed that the Stress Immunity subscale possesses a negative value $(\alpha=-.24)$, possibly due to a sudden increase of stress at the start of the experiment. Participants' total scores ranged from 116 to $143(\mathrm{M}=130.73, \mathrm{SD}=8.01)$, with higher scores indicating higher levels of psychopathic traits.

\section{State anxiety}

The State-Trait Anxiety Inventory - State Anxiety subscale (STAI Y1) is a self-report scale measuring state anxiety (Spielberger, Gorsuch, \& Lushene, 1970). The State Anxiety subscale consists of 20 items with answer options ranging from 1 ('Not at all') to 4 ('Very much so') with a possible score between 20 and 80 . The subscale assesses an individual's anxiety at a specific time or situation. Previous studies confirmed the scale's validity and that it has good internal consistency, with Cronbach's alpha ranging from $\alpha=.88$ to .93 (Fonseca Pedrero, Paino, Sierra Baigrie, Giráldez, \& Fernandez, 2012; Vigneau \& Cormier, 2008). The internal consistency for the STAI Y1 in the current sample at pretest and posttest were $\alpha=.83$ and $\alpha=.87$ respectively.

\section{Pain catastrophizing}

The Pain Catastrophizing Scale (PCS) is a self-report instrument assessing the extent to which an individual has catastrophizing thoughts (i.e.: 'I worry all the time about whether the pain will end') while under pain using a 13-item scale (Sullivan, Bishop, \& Pivik, 1995). The items are rated on a 5-point scale ranging from o ('Not at all') to 4 ('All the time'). The PCS is considered consistent with a previously reported alpha of $\alpha=.95$ (Osman et al., 2000). In the current study, Cronbach's alpha was $\alpha=.82$ at pretest and $\alpha=.86$ at posttest. 


\section{DURAND}

\section{Pain experience}

Three Numeric Rating Scales (NRS) were used to assess the experience of pain. The NRS ranged from 1 to 10 , and the participants were instructed to circle the number corresponding to their subjective perception of the item in question. Fear of pain was assessed with the question "How much do you fear the current pain you are experiencing?" Pain intensity was assessed with the question "How painful is the current pain you are experiencing?" The threat of pain was assessed with the question "How threatening is the pain you are currently experiencing?" Questions were administered between blocks (1 block being equal to 5 trials) when the pain stimulus was inactive. Items were formulated in terms of "current pain," which required the participant to take into consideration the whole block itself rather than a specific time point.

\section{Pain stimulus}

A Pathway model ATS thermode from the manufacturer Medoc Ltd., connected to the software E-Prime, was used. The active area of the thermode was $30 \times 30 \mathrm{~mm}$. The baseline temperature was set at the recommended value of $32^{\circ} \mathrm{C}$. During the experiment, the time until the participant started the pain stimulus was recorded via the space bar of a computer keyboard.

\section{Calibration}

All participants went through a calibration phase to ensure they could withstand the maximum temperature during the experiment, which was set at $47.7^{\circ} \mathrm{C}$. The thermode was attached to the ventral wrist of the dominant hand during both the calibration period and the experiment. The dominant hand was selected in order to maximize the recorded threat of pain, as individuals presumably feel a greater threat from something affecting their most functional hand. Calibration started at a baseline value of $32^{\circ} \mathrm{C}$ and increased by $1^{\circ} \mathrm{C}$ every second until the maximum of $47 \cdot 7^{\circ} \mathrm{C}$ was reached or when the participant pushed the stop button. This step was repeated three times, with a one-minute break between each session. The participant's personal maximum was set as the result obtained on the third calibration trial.

\section{Procedure}

At the beginning of the experiment, participants were invited to complete the PCS and the STAI-Y1. This step was followed by the calibration of the heat stimulus. All participants 


\section{DURAND}

achieved the maximum temperature of $47.7^{\circ} \mathrm{C}$ within three calibration trials. After resting for 10 minutes while filling in the PPI-SF, participants were placed in front of a monitor, ready to start the experiment.

The experiment consisted of 20 trials, which were divided into 4 blocks of five trials each. At the beginning of each trial, participants were instructed to press the space bar in order to see the temperature they would receive. Participants were led to believe that the temperature shown on screen was the temperature they would receive during the trial. The purpose of this deception was to further increase baseline levels of fear within all participants. The temperature on the screen started at $40^{\circ} \mathrm{C}$ and increased by $1^{\circ} \mathrm{C}$ after each trial. The real temperature received by participants through the thermode started at $43^{\circ} \mathrm{C}$ and increased by $0.25^{\circ} \mathrm{C}$ after each trial. Each trial started at baseline temperature $\left(32^{\circ} \mathrm{C}\right)$, and would increase in less than 2 seconds to the corresponding temperature. After the temperature was displayed, participants were given the choice to wait for a maximum of 10 seconds before receiving the heat stimulus. Alternatively, the participants could manually activate the pain stimulus before the end of the 10-second countdown by pressing the space bar of the computer keyboard. Once activated, the pain stimulus would last for a total of 7 seconds. After these 7 seconds, the temperature of the thermode would return to the baseline temperature, and participants would receive instructions to press the space bar when they were ready to see the temperature for the next trial on screen, followed by the next 10-second countdown. Between each block, participants were asked to fill in the three NRS to assess their experience of pain throughout the experiment. Participants completed the STAI Y1 and the PCS once again as posttest measures at the end of block 4. Participants' response times before starting the pain stimulus were recorded through the E-prime software after each trial.

\section{Statistical analyses}

The data were analyzed using SPSS version 21.00. Several types of statistical analyses were performed in order to answer the three hypotheses. First, PCS and STAY Y1 pre and post test scores were examined using a paired sample $t$ test in order to assess whether pain catastrophizing and anxiety levels would increase over time, as was predicted by the first hypothesis. Then, repeated multivariate analyses of variance (MANOVAs) were performed on the three NRS (fear of pain, pain intensity, and threat of pain) over the 4 time points in order to examine a potential increase of these variables over time. Post-hoc pairwise comparisons, adjusted via a Bonferroni correction for multiple testing, were performed to further test the first hypothesis in regard to an increase of fear of pain, pain intensity and threat of pain. A 
Bonferroni corrected repeated MANOVA was performed to test whether a preference to start the pain stimulus sooner as the experiment progressed would be observed. A Pearson correlation was performed on mean response time and fear-related variables to examine potential associations between lower response times and higher fear-related scores. Lastly, associations among psychopathic traits, fear-related variables, and mean response times were explored using Pearson bivariate correlations.

\section{RESULTS}

\section{Anxiety and pain catastrophizing levels over time}

Paired sample t-tests were performed on the PCS and STAI Y1 to examine whether anxiety and pain catastrophizing levels would increase by the end of the experiment. Although the mean scores at posttest $(M=9.80, S D=6.54$ and $M=30.13, S D=7.14)$ were lower than at pretest $(M$ $=13.13, \mathrm{SD}=7.09$ and $\mathrm{M}=32.40, \mathrm{SD}=6.34$ ) for both questionnaires, these differences were non-significant $(\mathrm{PCS}: \mathrm{t}(14)=1.92, \mathrm{p}=.076$, STAI Y1: $\mathrm{t}(14)=1.41, \mathrm{p}=.180)$.

\section{Fear of pain, pain intensity, and threat of pain over time}

Repeated MANOVAs were performed on the values obtained for the three NRS' (fear of pain, pain intensity and threat of pain) at the four time points to test whether fear of pain, pain intensity and threat of pain increased over time. A summary of these results can be found in Table 1. Several significant differences were found for all three scales. For the fear of pain scale, mean scores increased from 1.47 (o.74) after block 1 to 2.67 (1.59) after block 4, Wilks' $\Lambda=.40$, $(\mathrm{F}(3,12)=6.03, \mathrm{p}=.01)$. A within-subjects contrast test revealed a significant quadratic function $(\mathrm{F}(1,14)=10.03, \mathrm{p}=.007)$. Pairwise comparisons revealed that the mean score of fear of pain of block 4 was significantly higher than that of block $1(\mathrm{p}=.042)$, block $2(\mathrm{p}=.020)$, and block $3(\mathrm{p}=.003)$, indicating an overall increase of fear towards the end of the experiment. For the pain intensity scale, mean scores ranged from 1.87 (0.99) to 4.47 (2.30), increasing from block 1 to block 4 , Wilks' $\Lambda=.29,(F(3,12)=9.74, \mathrm{p}=.002)$. A within-subjects contrast test revealed a significant quadratic function $(F(1,14)=14.69, \mathrm{p}=.002)$. Pairwise comparisons did not identify a significant difference between block 1 and block 2. However, a significant increase was found between block 1 and block $3(\mathrm{p}=.020)$, between block 2 and block $3(\mathrm{p}=$ 
.o03), and between all other blocks (block 1 and 4 , block 2 and 4 , and block 3 and 4 ) (p<.oo1). For the threat of pain scale, mean scores ranged from 1.27 (0.46) to 2.87 (2.13), but were not significantly different from one another, Wilks' $\Lambda=.55,(\mathrm{~F}(3,12)=3.30, \mathrm{p}=.058)$. Therefore, no supplementary analysis was performed on the threat scale. Overall, these results partly support the first hypothesis, showing an increase in fear of pain and pain intensity over time during an increase in temperature.

Table 1. Mean score and standard deviation of the PCS, the STAI Y1, and the three Numeric Rating Scales at baseline and all four time points.

\begin{tabular}{llllll}
\hline & PCS & STAI Y1 & Fear of pain & Pain intensity & Threat \\
\hline Time o & $13.13(7.09)$ & $32.40(6.34)$ & & & \\
\hline Time 1 & & $1.47(0.74)$ & $1.87(0.99)$ & $1.27(0.46)$ \\
\hline Time 2 & & $1.47(0.74)$ & $2.07(1.16)$ & $1.40(0.63)$ \\
\hline Time 3 & & $1.80(1.14)$ & $3.00(1.46)$ & $1.80(0.94)$ \\
\hline Time 4 & $9.80(6.54)$ & $30.13(7.14)$ & $2.67(1.59)$ & $4.47(2.30)$ & $2.87(2.13)$ \\
\hline
\end{tabular}

Note. PCS = Pain Catastrophizing Scale; STAI Y1= State Trait Anxiety Inventory - State Anxiety subscale; Fear = Fear of pain; Pain = Pain intensity; Threat $=$ Threat of pain; Time $o=$ Baseline; Time 1 to $4=$ End of Block 1 to 4 .

\section{Change of response times over time}

Repeated MANOVAs were performed on the response times for each block to examine the second hypothesis, related to an expected difference over time to start the pain stimulus. Based on examination of the stem-and-leafs plots, two extreme values considered to be outliers were normalized by replacing their respective scores with the average of the corresponding block. Examination of the stem-and-leafs plots revealed that these outliers did not differ from the rest of the sample on other variables. Mean response time in seconds from block 1 to block 4 changed from $1.61 \mathrm{~ms}$ (0.59) to $0.96 \mathrm{~ms}(0.52)$, Wilks' $\Lambda=.34,(\mathrm{~F}(3,12)=7.78, \mathrm{p}=.004)$. As shown in Fig 1, mean response time throughout the experiment decreased in a cubic function, $(\mathrm{F}(1,14)=8.24, \mathrm{p}=.012)$. Pairwise comparisons with an applied Bonferroni correction revealed a significant difference between block 1 and $2(\mathrm{p}=.002)$, block 1 and $3(\mathrm{p}=.005)$, and block 1 and $4(\mathrm{p}=.001)$. Due to the rarity of cubic functions in psychological studies, these results should be interpreted with caution. 


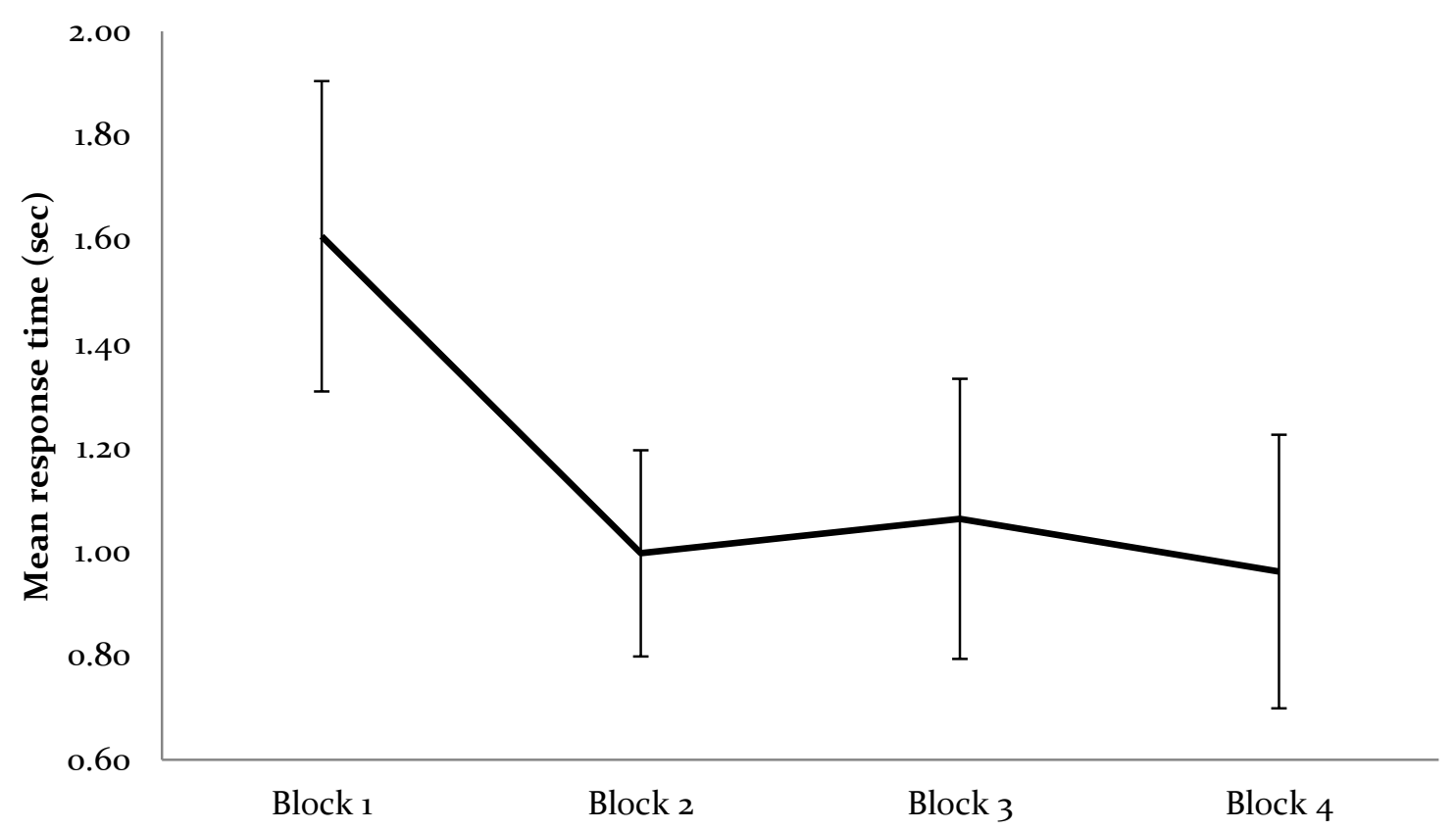

Figure 1. Mean response time in seconds across the four blocks with error bars at $95 \%$ confidence.

\section{Associations between response times and fear of pain, pain intensity and threat of pain}

A Pearson bivariate correlation was performed on mean response time and fear of pain, pain intensity and threat of pain in order to assess the potential existence of a relationship between high levels of fear of pain, pain intensity and threat of pain, and response times. The analysis revealed no significant correlation between any variables.

\section{Correlation with psychopathic traits}

Further analyses were performed to investigate the third hypothesis, which claimed a negative correlation between psychopathic traits and fear-related variables, as well as with mean response time to initiate the pain stimulus. A Pearson bivariate correlation was performed between the PPI-SF total, FD, and IA scores, along with all mean scores of fear-related variables and mean response time. While no significant correlations were found between the PPI-SF total score or the IA score and fear-related variables, positive correlations were found between FD and PCS scores at pretest $(\mathrm{r}=.53, \mathrm{p}=.044)$, indicating that higher levels of pain catastrophizing were associated with higher levels of FD. Furthermore, FD was also positively correlated with the sum of the painfulness NRS score across the four blocks $(r=.53, p=.044)$, 
and with the sum of the painfulness, fearfulness and threat NRS scores from block $1(\mathrm{r}=.61, \mathrm{p}=$ .017). No significant correlations were found between PPI-SF variables and mean response time.

\section{DISCUSSION}

This study sought to examine the potential for a heat pain stimulus to be used in a large scale experiment by examining how individuals react in terms of fear-related variables over time when exposed to an increasing heat stimulus. This experiment also examined potential fear conditioning through use of a heating device by assessing potential changes of preference over time towards immediate versus delayed onset of pain. Finally, correlations between psychopathic personality scores and fear-related variables, along with mean response time to initiate the pain stimulus, were examined to obtain preliminary results on the effectiveness of a heat pain device for a larger study on psychopaths.

Several conclusions can be drawn from the results of this pilot study. First, although posttest scores of the PCS and STAI Y1 were lower than the scores at pretest, these scores were not significantly different between time points. Indeed, pain catastrophizing and anxiety remained the same before and after the experiment. This lack of difference between pretest and posttest could be explained by the level of pain given throughout the experiment. It is possible that a heat pain device might not be painful enough to trigger an increase in pain catastrophizing or anxiety. These findings are inconsistent with the results obtained for the NRS ratings regarding fear of pain and pain intensity. While the threat of pain remained nonsignificant throughout the experiment, which could be explained by the safe nature of a laboratory environment in a university, fear of pain and pain intensity displayed significantly higher scores towards the end of the experiment than at the beginning. While there were no significant differences between each time point, it is possible that the heat-device was strong enough to elicit a change in subjective fear and pain levels towards the end of the experiment. However, the maximum averages obtained for fear of pain and pain intensity on a scale of 10 were 2.67 and 4.47 respectively, suggesting a weak increase overall. Despite their significance, the lack of differences between early time points, the differences between all time points for pain catastrophizing, anxiety and threat of pain, and the low average scores at the end of Block 


\section{DURAND}

4 suggest to reject the first hypothesis. These preliminary findings are not sufficiently conclusive to expect changes in the majority of fear-related variables in a larger scale study.

While encouraging, the present results also cannot support the second hypothesis, which expected a change over time in mean response time to initiate the pain stimulus. While a linear or quadratic regression would have been more promising, the significant cubic regression obtained is abnormal, as it supports a slow response time at the beginning and end of the experiment while displaying a fast response time during the middle of the experiment. Examination of the results demonstrates that only the response mean of block 1 differs from other blocks. It is possible that the higher mean response time in block 1 is due to a lack of habituation. After a few trials, participants may better understand the concept of pushing the space bar of the computer keyboard to initiate the pain, would explain the lack of difference between blocks 2, 3, and 4. This potential interpretation of mean response time is supported by the absence of any correlation between response time and fear-related variables.

The results obtained to confirm the third hypothesis failed to provide strong evidence of a correlation between psychopathic traits and the use of heat as a pain inducing stimulus. Indeed, no correlations were found between PPI-SF total score or IA and higher levels of fearrelated variables or different mean response time. Surprisingly, a few correlations were found between FD scores and higher levels of initial pain catastrophizing and fear-related variables, along with higher subjective painfulness. These results are inconsistent with the wellestablished notion of a lack of fear in FD (Fowles \& Dindo, 2009; Hale, Goldstein, Abramowitz, Calamari, \& Kosson, 2004; López, Poy, Patrick, \& Moltó, 2013). It is possible that these results stem from the limited number of participants. Furthermore, the negative alpha value found in the Stress Immunity subscale of the PPI-SF demonstrates that some individuals who started with low scores finished the questionnaires with high scores, and vice versa. Considering Stress Immunity is one of the three subscales of FD, it is possible that the responses reported by the participants were too inconsistent to provide a reliable FD score.

This pilot study possesses several limitations, starting with the low number of participants. Although this number is adequate to perform within-subject analysis on the whole sample, small correlations may only be detectable with a larger sample. However, considering the goal of the study, a small sample was necessary to critically evaluate the initial experimental design. This study was also limited by gender bias, as only men participated in the study. It is possible that the results could have differed by including a female sample. However, previous findings indicated a lack of correlation between psychopathic traits, fear of pain, and pain catastrophizing in a female sample (Caes et al., 2012). It may therefore be 


\section{DURAND}

necessary to focus the experiment on a male population before developing a mixed-gender study. Furthermore, in order to reduce possible acclimation effects, participants should be given a series of non-painful trials at the beginning to familiarize themselves with the procedure.

This study examined the effectiveness of a heat-pain device for triggering a psychological reaction in terms of pain catastrophizing, anxiety, fear of pain, pain intensity, and threat of pain. No differences were found in pain catastrophizing, anxiety and threat of pain throughout the experiment. While a few significant results were found in fear of pain and pain intensity over the course of the experiment, these results are not strong enough to validate the use of a heat pain stimulus. Furthermore, the difference in mean response time was only present in the first block, suggesting a habituation effect rather than fear conditioning over time. Finally, no correlations were found between fear-related variables and mean response time against PPI-SF total score, and inconsistent results were found on FD.

The aforementioned results suggest that several aspects of this experimental design need to be modified for future research. First, electrical pain stimuli should be prioritized over temperature pain stimuli, as electrical pain displays better outcomes in the field of psychopathy (Hare \& Thorvaldson, 1970; Hare, 1965, 1966; Miller et al., 2014). Second, the display of temperature on screen does not seem to be necessary and should be removed, as none of the participants reported to actually believe that the displayed temperature was the temperature they were receiving. Third, the experiment should avoid a low number of trials (n $=\mathbf{2 0}$ in the current experiment), and should instead implement a larger number of trials while instructing the participants to stop the experiment when their limit is reached. This method may cause more distress, especially in non-psychopaths, while ensuring a constant increase of pain in order to reduce the possibility of habituation. These modifications to the experimental design should be sufficient for a large-scale study assessing pain tolerance in psychopaths and non-psychopaths.

Overall, the present findings suggest that a heat pain stimulus may not be strong enough to trigger fear-related reactions in participants. Similar to the cold pressor previously used to assess tolerance of pain in psychopathic individuals, the heat pain stimulus fails to demarcate itself as a reliable pain-inducing instrument (Miller et al., 2014). It is therefore recommended to perform experiments assessing pain tolerance levels in psychopathic individuals by the use of electric shock, due to its reliability and easiness of use (Miller et al., 2014). 


\section{REFERENCES}

Berg, J. M., Smith, S. F., Watts, A. L., Ammirati, R., Green, S. E., \& Lilienfeld, S. O. (2013). Misconceptions regarding psychopathic personality: implications for clinical practice and research. Neuropsychiatry, 3, 63-74. http://doi.org/10.2217/npy.12.69

Caes, L., Uzieblo, K., Crombez, G., De Ruddere, L., Vervoort, T., \& Goubert, L. (2012). Negative emotional responses elicited by the anticipation of pain in others: Psychophysiological evidence. Journal of Pain, 13(5), 467-476. http://doi.org/10.1016/j.jpain.2012.02.003

Cale, E. M., \& Lilienfeld, S. O. (2006). Psychopathy factors and risk for aggressive behavior: A test of the "threatened egotism" hypothesis. Law and Human Behavior, 30(1), 51-74. http://doi.org/10.1007/s10979-006-9004-5

Fedora, O., \& Reddon, J. R. (1993). Psychopathic and nonpsychopathic inmates differ from normal controls in tolerance levels of electrical stimulation. Journal of Clinical Psychology, 49(3), $326-331$.

Fonseca Pedrero, E., Paino, M., Sierra Baigrie, S., Giráldez, S., \& Fernandez, J. (2012). Psychometric properties of the State-Trait Anxiety Inventory (STAI) in college students. Behavioral Psychology/Psicologia Conductual, 20(3), 547-561.

Fowles, D. C., \& Dindo, L. (2009). Temperament and psychopathy: A dual-pathway model. Current Directions in Psychological Science, 18(3), 179-183. http://doi.org/10.1111/j.14678721.2009.01632.x

Hale, L. R., Goldstein, D. S., Abramowitz, C. S., Calamari, J. E., \& Kosson, D. S. (2004). Psychopathy is related to negative affectivity but not to anxiety sensitivity. Behaviour Research and Therapy, 42(6), 697-710. http://doi.org/10.1016/S0005-7967(03)00192-X

Hare, R. D. (1965). Psychopathy, Fear Arousal and Anticipated Pain. Psychological Reports, 16, 499502. http://doi.org/10.2466/pr0.1965.16.2.499

Hare, R. D. (1966). Psychopathy and choice of immediate versus delayed punishment. Journal of Abnormal Psychology, 71, 25-29. http://doi.org/10.1037/h0022909

Hare, R. D. (1968). Detection Threshold for Electric Shock in Psychopaths. Journal of Abnormal Psychology, 73(3), 268-272.

Hare, R. D., \& Thorvaldson, S. A. (1970). Psychopathy and response to electrical stimulation. Journal of Abnormal Psychology, 76, 370-374. http://doi.org/10.1037/h0030276

Lilienfeld, S., \& Widows, M. (2005). Psychopathic personality inventory-revised: Professional manual. Lutz, FL: Psychological Assessment Resources.

López, R., Poy, R., Patrick, C. J., \& Moltó, J. (2013). Deficient fear conditioning and self-reported psychopathy: The role of fearless dominance. Psychophysiology, 50(2), 210-218. 


\section{DURAND}

http://doi.org/10.1111/j.1469-8986.2012.01493.x

Miller, J. D., Rausher, S., Hyatt, C. S., Maples, J., \& Zeichner, A. (2014). Examining the relations among pain tolerance, psychopathic traits, and violent and nonviolent antisocial behavior. Journal of Abnormal Psychology, 123(1), 205-13. http://doi.org/10.1037/a0035072

Mullins-Nelson, J. L., Salekin, R. T., \& Leistico, A.-M. R. (2006). Psychopathy, Empathy, and Perspective -Taking Ability in a Community Sample: Implications for the Successful Psychopathy Concept. International Journal of Forensic Mental Health, 5(2), 133-149. http://doi.org/10.1080/14999013.2006.10471238

Osman, A., Barrios, F. X., Gutierrez, P. M., Kopper, B. A., Merrifield, T., \& Grittmann, L. (2000). The Pain Catastrophizing Scale: Further Psychometric Evaluation with Adult Samples. Journal of Behavioral Medicine, 23(4), 351-365. http://doi.org/10.1023/A:1005548801037

Seibert, L. A., Miller, J. D., Few, L. R., Zeichner, A., \& Lynam, D. R. (2011). An examination of the structure of self-report psychopathy measures and their relations with general traits and externalizing behaviors. Personality Disorders: Theory, Research, and Treatment, 2(3), 193208. http://doi.org/10.1037/a0019232

Skeem, J. L., \& Cooke, D. J. (2010). Is criminal behavior a central component of psychopathy? Conceptual directions for resolving the debate. Psychological Assessment, 22(2), 433-445. http://doi.org/10.1037/a0008512

Spielberger, C. D., Gorsuch, R. L., \& Lushene, R. E. (1970). The State-Trait Anxiety Inventory. MANUAL, 1-23.

Sullivan, M., Bishop, S., \& Pivik, J. (1995). The pain catastrophizing scale: development and validation. Psychological Assessment, 7(4), 524-532. http://doi.org/10.1037/10403590.7.4.524

Tonnaer, F., Cima, M., Sijtsma, K., Uzieblo, K., \& Lilienfeld, S. O. (2013). Screening for psychopathy: Validation of the psychopathic personality inventory-short form with reference scores. Journal of Psychopathology and Behavioral Assessment, 35(2), 153-161. http://doi.org/10.1007/s10862-012-9333-2

Vigneau, F., \& Cormier, S. (2008). The factor structure of the State-Trait Anxiety Inventory: an alternative view. Journal of Personality Assessment, 90(3), 280-285. http://doi.org/10.1080/00223890701885027

Visser, B. A., Ashton, M. C., \& Pozzebon, J. A. (2012). Is Low Anxiety Part of the Psychopathy Construct? Journal of Personality, 80(3), 725-747. http://doi.org/10.1111/j.14676494.2011.00745.x 